International Mathematical Forum, Vol. 9, 2014, no. 19, 935 - 946

HIKARI Ltd, www.m-hikari.com

http://dx.doi.org/10.12988/imf.2014.4468

\title{
Warped Product Submanifolds of an S-Manifold
}

\author{
Reem A. Al-Ghefari and Salha K. Al-Tharwi \\ Department of Mathematics, Faculity of Science \\ King Abdulaziz University, P.O. Box 126300 \\ Jeddah 21352, Saudi Arabia
}

Copyright (c) 2014 Reem A. Al-Ghefari and Salha K. Al-Tharwi. This is an open access article distributed under the Creative Commons Attribution License, which permits unrestricted use, distribution, and reproduction in any medium, provided the original work is properly cited.

\begin{abstract}
In this paper we prove that there does not exist warped product of the type $N_{\theta} \times_{\lambda} N_{T}$ and $N_{T} \times_{\lambda} N_{\theta}$ of an S-manifold, where $N_{\theta}$ and $N_{T}$ are the slant and invariant submanifold of an S-manifold .We observe that only warped product of the type $N_{T} \times_{\lambda} N_{\perp}$ exist,with $N_{\perp}$ as a anti-invariant submanifolds of an S-manifold.Some basic results are discussed for the warped product of the type $N_{T} \times{ }_{\lambda} N_{\perp}$ and finally ,we prove an inequality for the squared norm of second fundamental form and equality case is also discussed.
\end{abstract}

Mathematics Subject Classification: 53C25, 53C40, 53C42, 53D15

Keywords: warped product, semi-slant, S-manifold

\section{Introduction}

The study of semi-invariant submanifold or contact CR-submanifolds of almost contact metric manifolds was initiated by A.Bejancu [3] .In particular ,semi-invariant submanifolds of different classes of almost contact metric manifolds have also been studied (c.f.,[4], [18]).Later N.Papaghuic[18] generalized the notion further and introduced semi-slant submanifolds of almost Hermition manifolds which includes the class of slant, holomorphic ,totally real and CR-submanifolds. J.L.Cabrerizo et al. [15] have studied semi-slant submanifolds of Sasakian manifolds. For manifolds with an $f$-structure ,D.E Blair [9] 
introduced S-manifolds as the analogue of Kaehler structure in almost complex case and of Sasakian structure in the almost contact case .

R.L.Bishop and B.O'Neil [20] introduced the notion of warped product manifolds. These manifolds are generaliztion of Riemannian product manifolds and occur naturally e.g.,surface of revolution is a warped product manifold $([16],[21])$.Due to wide applications of warped product submanifolds this becomes a fascinating and interesting topic for research and many articles are available in literature .CR-warped product was introduced by B.Y.chen $[7,8]$, he studied warped products CR-submanifolds in the setting of Kaehler manifolds and showed that there does not exist warped product CR-submanifolds of the form $M_{\perp} \times_{f} M_{T}$, therefore he considered warped product CR-submanifolds of the types $M_{T} \times_{f} M_{\perp}$ and established a relation ship between the warping fuction $f$ and the squared norm of the second fundamental form of the CR-warped product submanifolds in Kaehler manifolds .In the available literature,many geometers have studied warped products in the setting of almost contact metric manifolds (c.f. , [13], [21], [22]).

\section{Preliminaries}

Let $(\bar{M}, g)$ be a Riemannian manifold with $\operatorname{dim}(\bar{M})=2 n+s . \bar{M}$ is said to be an S-manifolds if there exists on $\bar{M}$ an $f$-structure $f$ of rank $2 \mathrm{n}$ and $\mathrm{s}$ global vector fields $\xi_{1}, \xi_{2}, \ldots, \xi_{s}$ (structure vector fields) such that (c.f., [9]).

(i) If $\eta_{1}, \eta_{2}, \ldots . \eta_{s}$ are the dual 1 -form of $\xi_{1}, \xi_{2}, \ldots, \xi_{s}$, then

$$
\begin{gathered}
f \xi_{\alpha}=0, \eta_{\alpha} \circ f=0, f^{2}=-\bar{I}+\sum_{\alpha=1}^{s} \eta_{\alpha} \otimes \xi_{\alpha} \\
g(f X, f Y)=g(X, Y)-\sum_{\alpha=1}^{s} \eta_{\alpha}(X) \eta_{\alpha}(Y)
\end{gathered}
$$

for any $X, Y \in T \bar{M}, \alpha=1,2, \ldots s$

ii) The $f$-structure $f$ is normal, that is

$$
[f, f]+2 \sum_{\alpha=1}^{s} \xi_{\alpha} \otimes d \eta_{\alpha}=0
$$


where $[f, f]$ is the Nijenhuis tensor of $f$.

iii) $\eta_{1} \wedge \eta_{2} \wedge \ldots, \wedge \eta_{s} \wedge\left(d \eta_{\alpha}\right)^{n} \neq 0$ and $d \eta_{1}=d \eta_{2}=\ldots=F$,for any $\alpha$, where $F$ is the fundamental 2 -form defined by

$$
F(X, Y)=g(X, f Y), \text { for any } X, Y \in T \bar{M}
$$

In the case $\mathrm{s}=1$, an $\mathrm{S}$-manifold is a Sasakian manifold . For $\mathrm{s} \geqslant 2$, examples of S-manifolds are given in $[9,10,11,12]$.

Moreover, for the Riemannian conection $\bar{\nabla}$ of g on an S-manifold $\bar{M}$, the following formulas were also proved in [9].

$$
\begin{gathered}
\left(\bar{\nabla}_{X} f\right) Y=\sum_{\alpha=1}^{s}\left\{g(X, Y) \xi_{\alpha}-X \eta_{\alpha}(Y)\right\} \\
\bar{\nabla}_{X} \xi_{\alpha}=-f X
\end{gathered}
$$

for any $X, Y \in T \bar{M}$, and $\alpha=1, \ldots, s$.

Let $\mathcal{L}$ denotes the distribution determined by $-f^{2}$ and $\mu$ the complementary distribution. $\mu$ is determined by $f^{2}+\bar{I}$ and spanned by $\xi_{1}, \xi_{2}, \ldots, \xi_{s}$. If $X \in \mathcal{L}$, then $\eta_{\alpha}(X)=0$ for any $\alpha$, and if $X \in \mu$, then $f X=0$.

Throughout, we denote by $\bar{M}$ an S-manifold , $M$ a submanifold of $\bar{M}$ with structure vector fields $\xi_{1}, \xi_{2}, \ldots, \xi_{s}$ tangent to $M . h$ and $A$ denote the second fundamental form and the shape opreator of the immersion of $M$ into $\bar{M}$ respectively .If $\nabla$ is the induced connection on $M$, the Gauss and Weingarten formulae of $M$ into $\bar{M}$ are then given as follows

$$
\begin{aligned}
& \bar{\nabla}_{X} Y=\nabla_{X} Y+h(X, Y) \\
& \bar{\nabla}_{X} V=-A_{V} X+\nabla_{X}^{\perp} V,
\end{aligned}
$$

for all vector fields $X, Y$ on $M$ and normal vector fields $V$ on $M, \nabla^{\perp}$ denotes the connection on the normal bundle $T M^{\perp}$ of $M . h$ and $A$ are related by

$$
g\left(A_{V} X, Y\right)=g(h(X, Y), V)
$$


where the induced Riemannian metric on $M$ is denoted by the same symbol $g$. Now , for any $x \in M, X \in T_{x} M$ and $V \in T_{x} M^{\perp}$,we put

$$
\begin{gathered}
f X=T X+N X \\
f V=t V+n V
\end{gathered}
$$

where $T X$ and $N X$ are the tangential and normal parts of $f X$ respectively and $t V$ and $n V$ are the tangential and normal parts of $f V$.

The covariant derivatives $\nabla T$ and $\nabla N$ are defined by

$$
\begin{aligned}
& \left(\nabla_{X} T\right) Y=\nabla_{X} T Y-T \nabla_{X} Y \\
& \left(\nabla_{X} N\right) Y=\nabla_{X}^{\perp} N Y-N \nabla_{X} Y
\end{aligned}
$$

Now, Let $M$ be an n-dimensional submanifold immersed in $\bar{M} \cdot M$ is said to be invariant submanifold if $\xi_{\alpha} \in T M$ for any $\alpha$ and $f X \in T M$, for any $X \in T M$ otherwise , $M$ is said to be anti-invariant submanifold if $f X \in T M^{\perp}$, for any $X \in T M$.

For a submanifold $M$ of an S-manifold $\bar{M}$ by equation (2.4),(2.5) and (2.8), we get

$$
\begin{gathered}
\nabla_{X} \xi_{\alpha}=-T X \\
h\left(X, \xi_{\alpha}\right)=-N X
\end{gathered}
$$

for each $X \in T M$.

Now using equations $(2.3),(2.5),(2.8),(2.10),(2.11)$ we get

$$
\left(\nabla_{X} T\right) Y=A_{N Y} X+\operatorname{th}(X, Y)+\sum_{\alpha=1}^{s} g(X, Y) \xi_{\alpha}-\sum_{\alpha=1}^{s} X \eta_{\alpha}(Y)
$$




$$
\left(\nabla_{X} N\right) Y=-h(X, T Y)+n h(X, Y)
$$

For any $x \in M$ and $X \in T_{X} M$ if the vectors $X$ and $\xi_{\alpha}(\alpha=1, \ldots, s)$ are linearly independent, the angle $\theta(X) \in\left[0, \frac{\pi}{2}\right]$ between $f X$ and $T_{X} M$ is well defined. If $\theta(X)$ does not depend on the choice of $x \in M$ and $X \in T_{X} M$,we say that $M$ is slant in $\bar{M}$. The constant angle $\theta$ is called the slant angle of $M$ in $\bar{M}$. If $\theta=\frac{\pi}{2}$ then $M$ is an anti-invariant submanifold and if $\theta=0 \mathrm{M}$ is invariant submanifold .

Carriazo et al.[1] give the following characterization for slant submanifold of S-manifolds.

Theorem 2.1 [1] Let $M$ be a submanifold of an $S$-manifold $\bar{M}$ such that $\xi_{\alpha} \in T M$.Then, $M$ is slant if and only if there exists a constant $\lambda \in[0,1]$ such that

$$
T^{2}=-\lambda \sum_{\alpha=1}^{s}\left(\bar{I}-\eta_{\alpha} \otimes \xi_{\alpha}\right)
$$

Furthermore, in such case, if $\theta$ is the slant angle of $M$, then it verifies that $\lambda=\cos ^{2} \theta$.

Now, we have the following Corollary,which can be proved directly by (2.16).

Corollary 2.1 Let $M$ be a slant submanifold of an $S$-manifold $\bar{M}$ such that $\xi_{\alpha} \in T M$.Then,

$$
\begin{aligned}
& g(T X, T Y)=\cos ^{2} \theta\left[g(X, Y)-\sum_{\alpha=1}^{s} \eta_{\alpha}(X) \eta_{\alpha}(Y)\right] \\
& g(N X, N Y)=\sin ^{2} \theta\left[g(X, Y)-\sum_{\alpha=1}^{s} \eta_{\alpha}(X) \eta_{\alpha}(Y)\right]
\end{aligned}
$$

for any $X, Y \in T M$.

A semi-slant submanifold $M$ of an almost contact metric manifold $\bar{M}$ is a submanifold which contains two orthogonal complementary distribution $D$ 
and $D_{\theta}$, such that $D$ is invariant under $f$ and $D_{\theta}$ is slant with slant angle $\theta \neq 0$, i.e , $f D=D$ and $f Z$ makes a constant angle $\theta$ with $T M$ for each $Z \in D_{\theta}$.In particular if $\theta=\frac{\pi}{2}$, then semi-slant submanifolds reduced to CR-submanifold defined in [3].For a semi-slant submanifold $M$ of an S-manifold, we have

$$
T M=D \oplus D_{\theta} \oplus\langle\xi\rangle
$$

The orthogonal complement of $N D_{\theta}$ in the normal bundle $T M^{\perp}$, is an invariant subbundle of $T M^{\perp}$ and is denoted by $\mu$.Thus, we have

$$
T M^{\perp}=N D_{\theta} \oplus \mu
$$

A semi-slant submanifold $M$ is called a semi-slant product if the distributions $D$ and $D_{\theta}$ are involutive and parallel on $M$. In this case $M$ is foliated by the leaves of these distributions.

As a generalization of the product manifolds and in particular of a semislant product submanifold, one can consider warped product of manifolds which are defined as

Definition 2.1 Let $\left(B, g_{B}\right)$ and $\left(F, g_{F}\right)$ be two Riemannian manifolds with Riemannian metric $g_{B}$ and $g_{F}$ respectively and $\lambda$ positive differentiable function on $B$.The warped product of $B$ and $F$ is the Riemannian manifold $(B \times F, g)$, where

$$
g=g_{B}+\lambda^{2} g_{F}
$$

For a warped product manifold $N_{1} \times_{\lambda} N_{2}$, we denote by $D_{1}$ and $D_{2}$ the distributions defined by the vectors tangent to the leaves and fibers respectively

In other words, $D_{1}$ is obtained by the tangent vectors of $N_{1}$ via the horizontal lift and $D_{2}$ is obtained by the tangent vectors of $N_{2}$ via vertical lift .In case of semi-slant warped product submanifolds $D_{1}$ and $D_{2}$ are replaced by $D$ and $D_{\theta}$ respectively .

The warped product manifold $(B \times F, g)$ is denoted by $B \times{ }_{\lambda} F$.If $X$ is the tangent vector field to $M=B \times_{\lambda} F$ at $(p, q)$ then

$$
\|X\|^{2}=\left\|d \pi_{1} X\right\|^{2}+\lambda^{2}(p)\left\|d \pi_{2} X\right\|^{2} .
$$

R.L.Bishop and B.O'Neill [20] proved the following

Theorem 2.2 Let $M=B \times_{\lambda} F$ be a warped product manifolds. If $X, Y \in T B$ and $V, W \in T F$ then 
(i) $\nabla_{X} Y \in T B$

(ii) $\nabla_{X} V=\nabla_{V} X=\left(\frac{X \lambda}{\lambda}\right) V$,

(iii) $\nabla_{V} W=\frac{-g(V, W)}{\lambda} \nabla \lambda$.

$\nabla \lambda$ is the gradient of $\lambda$ and is defined as

$$
g(\nabla \lambda, X)=X \lambda
$$

for all $X \in T M$.

Corollary 2.2 On a warped product manifold $M=N_{1} \times_{\lambda} N_{2}$, the following statements hold

(i) $N_{1}$ is totally geodesic in $M$,

(ii) $\mathrm{N}_{2}$ is totally umbilical in $M$,

\section{Warped Product Submanifolds of S-manifolds}

Let $\bar{M}$ be an S-manifold.Throughout this section,we denote by $N_{T}$ an invariant submanifold of $\bar{M}$ and $N_{\theta}$ a slant submanifold of $\bar{M}$, with slant angle $\theta$.

Matsumoto and Mihai [17] proved the following theorem .

Theorem 3.1 If $N_{1} \times_{\lambda} N_{2}$ is a warped product submanifold of a Sasakian manifold $\bar{M}$, where $N_{1}$ and $N_{2}$ are any submanifold of a Sasakian manifold $\bar{M}$ with $\xi$ tangential to $N_{2}$. Then $M$ is a Riemannian product.

On the line of Theorem 3.1 , we can prove the following theorem

Theorem 3.2 If $N_{1} \times_{\lambda} N_{2}$ is a warped product submanifold of an S-manifold $\bar{M}$, where $N_{1}$ and $N_{2}$ are any submanifold of an $S$-manifold $\bar{M}$ with $\xi_{\alpha}$ tangential to $N_{2}$. Then $M$ is a Riemannian product .

Hence we can consider only nontrivial semi-slant warped product submanifolds as $N_{\theta} \times_{\lambda} N_{T}$ and $N_{T} \times_{\lambda} N_{\theta}$ with $\xi_{1}, \xi_{2}, \ldots, \xi_{s}$ tangential to $N_{\theta}$ and $N_{T}$ respectively .If $\theta=\frac{\pi}{2}$ these warped products are known as warped product contact CR-submanifolds and contact CR-warped product submanifolds respectively .

Theorem 3.3. Let $\bar{M}$ be a $(2 m+s)$-dimensional $S$-manifold. Then there do not exists warped product submanifolds $N_{\theta} \times_{\lambda} N_{T}$ on $\bar{M}$ such that $N_{\theta}$ is slant 
submanifold tangent to $\xi_{1}, \xi_{2}, \ldots, \xi_{s}$ and $N_{T}$ is an invariant submanifold of $\bar{M}$.

Proof. For warped product submanifold $N_{\theta} \times_{\lambda} N_{T}$ of $\bar{M}$ with $\xi_{\alpha}$ tangential to $N_{\theta}$. Then by Theorem 2.2

$$
\nabla_{X} Z=\nabla_{Z} X=Z \ln \lambda X
$$

for any $X \in T N_{T}$ and $Z \in T N_{\theta}$.In particular for $Z=\xi_{\alpha}$, from equations (2.12) and (3.1) ,

$$
\xi_{\alpha} \ln \lambda X=0,
$$

this mean $\xi_{\alpha} \ln \lambda=0$ i.e., $\lambda$ is constant, hence warped product does not exists.

On the same line of Theorem 3.3 of [22], we can prove the following theorem.

Theorem 3.4 There does not exists a warped product semi-slant submanifold of the type $N_{T} \times_{\lambda} N_{\theta}$ in an $S$-manifold other than a contact CR-warped submanifold.

The above theorem motivates us to study the warped product of the type $N_{T} \times_{\lambda} N_{\perp}$ in S-manifolds .

Let $M=N_{T} \times_{\lambda} N_{\perp}$ be a contact CR-warped product submanifold of an S-manifold $\bar{M}$. In view of decomposition (2.17), we may write

$$
h(X, Y)=h_{f D^{\perp}}(X, Y)+h_{\mu}(X, Y)
$$

for each $X, Y \in T M$, where $h_{f D^{\perp}}(X, Y) \in f D^{\perp}$ and $h_{\mu}(X, Y) \in \mu$.If $\left\{e_{1}, e_{2}, \ldots, e_{n}\right\}$ be a local orthonormal frame of vector fields on $M$ then we define

$$
\|h\|^{2}=\sum_{i, j=1}^{n} g\left(h\left(e_{i}, e_{j}\right), h\left(e_{i}, e_{j}\right)\right) .
$$

Now we have the following proposition for the warped product of the type $N_{T} \times{ }_{\lambda} N_{\perp}$.

Proposition 3.1. Let $M=N_{T} \times_{\lambda} N_{\perp}$ be a contact CR-warped product submanifold of an $S$-manifold $\bar{M}$. Then

(i) $h_{f D^{\perp}}(X, Z)=-f X \ln \lambda f Z-\sum_{\alpha=1}^{s} \eta_{\alpha}(X) f Z$,

(ii) $g(h(f X, Z), f W)=X \ln \lambda g(Z, W)$,

(iii) $g(h(f X, Z), f h(X, Z))=\|h(X, Z)\|^{2}$,

for any $X \in T N_{T}$ and $Z, W \in T N_{\perp}$.

Proof. By Gauss formula

$$
h(f X, Z)=\left(\bar{\nabla}_{Z} f\right) X+f \nabla_{Z} X+f h(X, Z)-\nabla_{Z} f X,
$$


Using equations (2.3),(2.5) and (3.1), we have

$$
h(f X, Z)=-\sum_{\alpha=1}^{s} Z \eta_{\alpha}(X)+X \ln \lambda f Z+f h(X, Z)-f X \ln \lambda Z
$$

Comparing tangential parts on above equation, we get

$$
\sum_{\alpha=1}^{s} Z \eta_{\alpha}(X)=f h_{f D^{\perp}}(X, Z)-f X \ln \lambda Z
$$

Or

$$
f h_{f D^{\perp}}(X, Z)=f X \ln \lambda Z+\sum_{\alpha=1}^{s} Z \eta_{\alpha}(X)
$$

taking inner product with $W \in D^{\perp}$ on both side, we find

$$
-g(h(X, Z), f W)=f X \ln \lambda g(Z, W)+\sum_{\alpha=1}^{s} \eta_{\alpha}(X) g(Z, W),
$$

Or

$$
h_{f D^{\perp}}(X, Z)=-f X \ln \lambda f Z-\sum_{\alpha=1}^{s} \eta_{\alpha}(X) f Z,
$$

Which proves the part (i) of the proposition .

Now on comparing the normal parts

$$
h(f X, Z)=X \ln \lambda f Z+f h_{\mu}(X, Z)
$$

Or

$$
h(f X, Z)-f h_{\mu}(X, Z)=X \ln \lambda f Z,
$$

Taking inner product with $f W$, the above equation yields

$$
g(h(f X, Z), f W)=X \ln \lambda g(Z, W)
$$

Again taking inner product with $f h(X, Z)$ in $(3.3)$, we find

$$
g(h(f X, Z), f h(X, Z))=\|h(X, Z)\|^{2},
$$

which is the part (iii) of the proposition .

For contact CR-warped product submanifold $M=N_{T} \times_{\lambda} N_{\perp}$ of an Smanifold $\bar{M}$, we have the following theorem. 
Theorem 3.5 Let $M=N_{T} \times{ }_{\lambda} N_{\perp}$ be a contact $C R$-warped product submanifold of an $S$-manifold $\bar{M}$ then

(i) The squared norm of the second fundamental form satisfies

$$
\|h\|^{2} \geqslant 2 q\|\nabla \ln \lambda\|^{2}+q s
$$

where $\nabla \ln \lambda$ is the gradient of $\ln \lambda$ and $q$ is the dimension of anti-invariant distribution .

(ii) The equality holds if $h(D, D)=0$ and $h\left(D^{\perp}, D^{\perp}\right)=0$.

Proof. Let $\left\{\xi_{1}, \xi_{2}, \ldots, \xi_{s}, X_{1}, X_{2}, \ldots, X_{P}, X_{P+1}=f X_{1}, \ldots, X_{2 P}=f X_{P}\right\}$ be a local orthonormal frame of vector fields on $N_{T}$ and $\left\{Z_{1}, Z_{2}, \ldots, Z_{q}\right\}$ be the local orthonormal frame of vector fields on $N_{\perp}$. Then by the definition of squared norm of the mean curvature vector

$$
\begin{gathered}
\|h\|^{2}=\sum_{i, j=1}^{2 P} g\left(h\left(X_{i}, X_{j}\right), h\left(X_{i}, X_{j}\right)\right)+\sum_{i=1}^{2 p} \sum_{r=1}^{q} g\left(h\left(X_{i}, Z_{r}\right), h\left(X_{i}, Z_{r}\right)\right) \\
+\sum_{r, s=1}^{q} g\left(h\left(Z_{r}, Z_{s}\right), h\left(Z_{r}, Z_{s}\right)\right)+\sum_{i=1}^{2 p} \sum_{\alpha=1}^{s} g\left(\left(h\left(X_{i}, \xi_{\alpha}\right), h\left(X_{i}, \xi_{\alpha}\right)\right)\right. \\
+\sum_{r=1}^{q} \sum_{\alpha=1}^{s} g\left(h\left(Z_{r}, \xi_{\alpha}\right), h\left(Z_{r}, \xi_{\alpha}\right)\right)
\end{gathered}
$$

Using equation (2.12), the above equation provides us the following inequality

$$
\|h\|^{2} \geqslant \sum_{i=1}^{2 p} \sum_{r=1}^{q} g\left(h\left(X_{i}, Z_{r}\right), h\left(X_{i}, Z_{r}\right)\right)+\sum_{r=1}^{q} \sum_{\alpha=1}^{s} g\left(h\left(Z_{r}, \xi_{\alpha}\right), h\left(Z_{r}, \xi_{\alpha}\right)\right)
$$

On using part (i) of the proposition (3.1) and equation (3.3), the above inequality takes the form

$$
\|h\|^{2} \geqslant \sum_{i=1}^{2 p} \sum_{r=1}^{q}\left(f X_{i} \ln \lambda\right)^{2} g\left(Z_{r}, Z_{r}\right)+q s
$$

Or

$$
\|h\|^{2} \geqslant 2 q\|\nabla \ln \lambda\|^{2}+q s,
$$

Which is a required inequality .

It is evident from (3.4), that if inequality holds identically, then $h(D, D)=$ $0, h\left(D^{\perp}, D^{\perp}\right)=0$. 


\section{References}

[1] A.Carriazo , L.M.Fernandez,M.B.Hans-Uber,Some slant submanifolds of Smanifolds,Acta math .Hungar.,108 (2005),1-12.

[2] A.Lotta, Slant Submanifolds in Contact Geometry,Bull.Math.Soc.Roum.,39 (1996),183-198.

[3] A.Bejancu,N.Papaghuic,Semi-invariant submanifolds of Sasakian manifolds, Anale Stiin.ale.Univ.Al.I.Cuza Iasi,Tom XXVII S.I.A.,(27)(1981),163-170.

[4] A.Alghanemi,CR-Submanifolds of an S-manifold,Turk J Math.,32 (2008),141154.

[5] Chen B Y,Slant Immersions,Bull Aust Math Soc,41(1990). 135-147.

[6] B.Y.Chen, CR-submanifolds of Kaehler manifold I, J. Differential Geometry 16 (1981), 305-323.

[7] B.Y.Chen,Geometry of warped product CR-submanifolds in Kaehler manifolds I, Monatsh. Math.133(2001), 177-195.

[8] B.Y.Chen,Geometry of warped product CR-submanifolds in Kaehler manifolds II, Monatsh. Math.134(2001),103-119.

[9] D.E.Blair, Geometry of manifolds with structural group $U(n) \times O(s)$, J. Differential Geom., 4(1970), 155-167.

[10] D.E.Blair ,On a generalization of the Hopf fibration,Ann.Stiint.Univ.Al.I Cuza Iasi,17(1) (1971),171177.

[11] D.E.Blair ,G.D.Ludden and K.Yano,Differential geometric structures on principal toroidal bundles,Trans.Amer.Math.Soc.,181(1973),175184.

[12] I.Hasegawa,Y.Okuyama and T.Abe,On p-th Sasakian manifolds, J.Hokkaido Univ.Ed.Sect.,II A 37 (1) (1986),116.

[13] I.Hasegawa and I.Mihai,Contact CR-warped product submanifolds in Sasakian manifolds, Geom.Dedicate,102 (2003), 143-150.

[14] J.L.Cabrerizo,L.M.Fernandez and M.Fernandez, A classification of totally f-umbilical submanifolds of an S-manifold, Soochow J.Math., 18 (2) (1992), 
$211-221$.

[15]J.L.Cabrerizo,A.Carriazo,L.M.Fernandez and M.Fernandez, Semi-slant Submanifolds in Sasakian Manifolds,Geometriae Dedicata, 78 (1999) ,183-199.

[16] J.K.Beem, P.E.Ehrlich, K.Easley, Global Lorentzian geometry , Marcel Dekker, New York 1996.

[17] K.Matsumoto,I.Mihai,Warped product submanifolds in Sasakian space form, SUT J.Math., 38,2(2002), 135-144.

[18] L.M.Fernandez,CR-products of S-manifolds,Portugal. Mat., 47 (2) (1990), 167181.

[19] N.Papaghuic, Semi-slant Submanifolds of Kaehlerian Manifold, An. Stiint. Univ. Iasi, $9\left(f_{1}\right)(1994)$, 55-61.

[20] R.L.Bishop, B.O’Neill, Manifolds of Negative curvature , Trans. Amer. Math. Soc., 145 (1965), 1-49.

[21] S.T.Hong, Warped products and black holes, Nuovo Cim. J.B 120 (2005), 1227-1234.

[22] Falleh R. Al-Solamy, Viqar Azam Khan, Warped product semi-slant submanifolds of a Sasakian manifold , Serdica Math.J.,34(2008), 597-606.

Received: April 11, 2014 